Published in final edited form as:

J Matern Fetal Neonatal Med. 2013 May ; 26(7): 647-653. doi:10.3109/14767058.2012.746297.

\title{
Placental villous hypermaturation is associated with idiopathic preterm birth
}

\author{
Terry K. Morgan ${ }^{1,2}$, Jorge E. Tolosa ${ }^{1}$, Lisa Mele ${ }^{3}$, Ronald J. Wapner ${ }^{4}$, Catherine Y. Spong ${ }^{5}$, \\ Yoram Sorokin ${ }^{6}$, Donald J. Dudley ${ }^{7}$, Alan M. Peaceman ${ }^{8}$, Brian M. Mercer ${ }^{9}$, John M. \\ Thorp $^{10}$, Mary Jo O'Sullivan ${ }^{11}$, Susan M. Ramin ${ }^{12}$, Dwight J. Rouse ${ }^{13}$, and Baha Sibai ${ }^{14}$ for \\ the Eunice Kennedy Shriver National Institute of Child Health and Human Development \\ Maternal-Fetal Medicine Units Network \\ ${ }^{1}$ Departments of Obstetrics \& Gynecology, Oregon Health \& Science University, Portland, OR, \\ USA \\ ${ }^{2}$ Departments of Pathology, Oregon Health \& Science University, Portland, OR, USA \\ ${ }^{3}$ Biostsistics Center, George Washington University, Washington, DC, USA \\ ${ }^{4}$ Department of Obstetrics and Gynecology, Columbia University, New York, USA \\ ${ }^{5} \mathrm{NICHD}$, Bethesda, MD, USA \\ ${ }^{6}$ Department of Obstetrics and Gynecology, Wayne State University, Detroit, MI, USA \\ ${ }^{7}$ Department of Obstetrics and Gynecology, University of Utah, Salt Lake City, UT, USA \\ ${ }^{8}$ Department of Obstetrics and Gynecology, Northwestern University, Chicago, IL, USA \\ ${ }^{9}$ Department of Obstetrics and Gynecology, Case Western Reserve University- MetroHealth \\ Medical Center, Cleveland, $\mathrm{OH}$, USA \\ ${ }^{10}$ Department of Obstetrics and Gynecology, University of North Carolina at Chapel Hill, Chapel \\ Hill, NC, USA \\ ${ }^{11}$ Department of Obstetrics and Gynecology, University of Miami, Miami, FL, USA \\ ${ }^{12}$ Department of Obstetrics and Gynecology, The University of Texas Health Science Center at \\ Houston, Houston, TX, USA \\ ${ }^{13}$ Department of Obstetrics and Gynecology, University of Alabama at Birmingham, Birmingham, \\ AL, USA \\ ${ }^{14}$ Department of Obstetrics and Gynecology, University of Tennessee, Memphis, TN, USA
}

\section{Abstract}

(C) 2012 Informa UK Ltd.

Address for correspondence: Terry K. Morgan, MD, Ph.D., Departments of Pathology and Obstetrics \& Gynecology, Oregon Health \& Science University, 3181 SW Sam Jackson Park Road, Mail Code L471, Portland, OR 97239, USA. Tel: (503) 494-6782. morgante@ohsu.edu.

Declaration of Interest

The authors report no declarations of interest other than the following finanacial support. 
Objective-Pregnancy complications such as intra-amniotic infection, preeclampsia, and fetal intrauterine growth restriction (IUGR) account for most cases of preterm birth (PTB), but many spontaneous PTB cases do not have a clear etiology. We hypothesize that placental insufficiency may be a potential cause of idiopathic PTB.

Methods-Secondary analysis of 82 placental samples from women with PTB obtained from a multicenter trial of repeat versus single antenatal corticosteroids. Samples were centrally reviewed by a single placental pathologist masked to clinical outcomes. The histopathologic criterion for infection was the presence of acute chorioamnionitis defined as neutrophils marginating into the chorionic plate. Placental villous hypermaturation $(\mathrm{PVH})$ was defined as a predominance of terminal villi (similar to term placenta) with extensive syncytial knotting. Idiopathic PTB comprised a group without another known etiology such as preeclampsia, IUGR or infection.

Results-Acute chorioamnionitis was observed in 33/82 (40\%) cases. Other known causes of PTB were reported in 18/82 (22\%). The remaining 31/82 (38\%) were idiopathic. The frequency of PVH in idiopathic PTB $(26 / 31=84 \%)$ was similar to cases with IUGR or preeclampsia (16/ $18=89 \%$ ), but significantly more common than PVH in the group with acute chorioamnionitis $(10 / 33=30 \%)(p<0.001)$.

Conclusions-PVH, which is a histologic marker of relative placental insufficiency, is a common finding in idiopathic PTB.

\section{Keywords}

Chorioamnionitis; histology; idiopathic preterm labor; placenta; Tenney-Parker change

\section{Introduction}

Spontaneous preterm birth (PTB) is a leading cause of perinatal morbidity and mortality [1]. Although some preterm deliveries are associated with intra-amniotic infection [2-4], many spontaneous PTB cases lack a defined etiology (idiopathic).

The causes of spontaneous PTB are likely multifactorial [5,6]. Depending on the gestational age studied and techniques utilized, evidence of intra-amniotic infection is reported in 15$60 \%$ of the cases [2-4]. This wide range may be one reason that treatments targeted against infection are sometimes ineffective [7]. One underappreciated cause of idiopathic PTB may be placental insufficiency. Placental insufficiency is a clinical term [8] essentially used to describe our ignorance [9], but placental findings appear to be related to oxidative stress [10-12]. The underlying cause may be abnormal uterine spiral artery remodeling [13-22], leading to abnormal uteroplacental blood flow [10,23,24].

Placental insufficiency is associated with characteristic placental pathology [25-30]. Socalled pre-placental injury is related to abnormal uteroplacental blood flow and is diagnosed by the presence of reduced placental weight, placental infarctions, accelerated chorionic villous maturation, conspicuous syncytial knotting (Tenney-Parker change) and perivillous fibrin deposition $[8,12,25-27,30]$. The combination of accelerated villous maturation [12,30] and Tenney-Parker changes [12,25-27], which we call placental villous hypermaturation $(\mathrm{PVH})$, may be used as histologic evidence of placental insufficiency, because it is 
associated with severe preeclampsia $[25,26]$ and late onset intrauterine growth restriction (IUGR) [26]. In contrast, severe early-onset IUGR shows a different histologic pattern, socalled distal villous hypomaturation [12,29], which is a developmental deficit in terminal villous differentiation [12]. Nonetheless, studies performed by specialized placental pathologists suggest the overall histologic impression of placental insufficiency is reproducible (kappa statistic 0.68) [29]; and, although pathologists may have difficulty precisely dating the gestational age of the placenta [31], they can reasonably agree on whether the placenta has preterm or term villous maturation (kappa statistic 0.58) [32].

We hypothesize that similar to preeclampsia and late onset IUGR, idiopathic spontaneous PTB may also be associated with placental histologic features of insufficiency. To test this hypothesis, cases of intra-amniotic infection should be identified and analyzed as a separate group. Ideally, microbiologic cultures and PCR would be used to test amnionic fluid for infection [2,4], but in the absence of this gold standard, studies may employ placental histology. Placental histology has a high negative predictive value (97\%) and reasonable positive predictive value (79\%) for intra-amniotic infection compared with fluid cultures [4]. Therefore, in the absence of acute chorioamnionitis, intra-amniotic infection is unlikely [4]. In this study, we compared the frequency of PVH in idiopathic PTB with the frequency in a group of cases positive for histologic evidence of intra-amniotic infection and a third group of cases known to cause PTB (i.e. preeclampsia and IUGR).

\section{Methods}

\section{Patient samples}

This study was approved by the Oregon Health \& Science University Institutional Review Board. We performed a retrospective secondary analysis of placental samples collected from women with intact membranes who presented with an increased risk for PTB. Cases were collected during the NICHD MFMU Network multicenter trial to study the effects of single versus weekly courses of antenatal corticosteroids in a tightly controlled randomized fashion [33]. These women gave ethical consent for the use of their placental samples and clinical data. Placental histologic sections were available from 82 cases of spontaneous PTB. The effect of single versus multiple courses of antenatal corticosteroids had previously been shown in this cohort to have no effect on placental histology [34]. However, we controlled for this confounder in our multivariate analysis, because others have suggested steroids may cause histologic features similar to PVH [35]. Idiopathic PTB was defined as cases not associated with other known causes of PTB, such as histologic evidence of intra-amniotic infection (including 16 cases of preterm premature rupture of membranes (PPROM) with acute chorioamnionitis), or a third eclectic group composed of preeclampsia ( $n=2)$, IUGR $(n=6)$, abruption $(n=1)$, preterm PPROM negative for acute chorioamnionitis $(n=7)$ and insulin-dependent diabetes $(n=2)$. These exclusion criteria yielded 31 cases of idiopathic PTB, 33 cases associated with histologic evidence of intra-amniotic infection and 18 cases with other known causes of PTB without infection that are often associated with placental insufficiency. 


\section{Placental histologic examination}

Multiple histologic sections were available for each case, including two sections of the umbilical cord, a section of placental membranes, and at least two representative sections of placental parenchyma. Gross placental weights were not available for analysis. Histologic examination of the 82 cases was performed by a single placental pathologist (TKM) while blinded to gestational age, steroid treatment group and clinical outcomes. Routine hematoxylin \& eosin (H\&E)- stained sections were scored for pathologic findings defined based on descriptions in Pathology of the Human Placenta by Drs Benirschke and Kaufmann [30] and numerous peer-reviewed studies [18-29]. Briefly, placental sections were scored as positive for PVH if the finding were diffusely present (multiple $10 \times$ objective fields in multiple histologic sections) in preterm samples ( $<37$ weeks) and characterized by a "predominance of terminal villi. . . usually found in pregnancy at 38-41 weeks" [30] with conspicuous syncytial knotting (Figure 1E). Distal villous hypoplasia [29] was not observed in our cohort, perhaps because there were no cases of severe early onset IUGR [12]. Sections were scored as positive for acute chorioamnionitis if there was at least "acute chorionitis" (so-called grade 1 chorioamnionitis (Figure 1)), which requires multiple clusters of maternal neutrophils marginating into the placental chorionic plate [4]. Only cases negative for acute chorioamnionitis were considered negative for infection.

\section{Statistical analysis}

Statistical analysis was conducted using SAS software (SAS Institute, Cary, NC). Categorical variables were compared using the chi-square or Fisher's Exact tests. Continuous variables were compared using the Wilcoxon Rank-Sum test or Kruskall-Wallis test. Multivariate analysis was performed on PTB cases to examine the relationship between PVH and idiopathic PTB, compared with acute chorioamnionitis, and other known causes of PTB. Covariates included in the model were single versus repeat antenatal steroids, parity, and gestational age at delivery. A nominal two-sided $p<0.05$ was considered significant.

\section{Results}

The mean gestational age was 33 weeks \pm 3.0 [range 26-37 weeks] and was not significantly different between the three groups, although cases with acute chorioamnionitis did deliver about 2 weeks earlier than the other two groups. Maternal race was evenly distributed between pregnancy outcome groups (approximately 43\% black, 39\% white, 18\% Hispanic) and there was no difference in the distribution of steroid treatment number.

Histologic evidence of acute chorioamnionitis was identified in 33/82 (40\%) cases. Inflammation involving the amnion was observed in 24/33 and funisitis was seen in 25/33 of these cases. Acute chorioamnionitis was diagnosed in 16/23 (70\%) cases of PPROM. The remaining seven negative PPROM cases were not considered idiopathic, but instead were included in the group of PTB cases often associated with placental insufficiency (e.g. preeclampsia, IUGR, abruption, diabetes). This group comprised 18/82 (22\%) preterm deliveries in our study. Therefore, our study isolated 31/82 (38\%) cases of idiopathic spontaneous PTB. 
The frequency of PVH in the idiopathic PTB group (26/31=84\%) was significantly greater than in cases with acute chorioamnionitis $(10 / 33=30 \%)(p<0.001)$. This association was independent of the infection diagnostic category (funisitis, acute chorionitis [grade 1] [4], and acute amnionitis). The frequency of PVH in the idiopathic group was similar to that observed in our preeclampsia/IUGR group $(16 / 18=89 \%)$ (Table 1). There were no significant associations between PVH and parity, (nulligravida PVH frequency [55\%], compared with multigravida [67\%]; $p=0.31$ ), or corticosteroid administration (single course PVH frequency [58\%], compared with repeat courses [68\%]; $p=0.33$ ). Multivariate analysis did not reveal a statistically significant relationship between $\mathrm{PVH}$ and race, or gestational age, but there appeared to be an increased frequency of acute chorioamnionitis delivering before 34 weeks (Figure 2).

\section{Discussion}

We report that PVH is common in idiopathic spontaneous PTB similar to pregnancy complications like preeclampsia and IUGR, which are thought to be associated with placental insufficiency. This placental finding is significantly less common in PTB cases positive for histologic evidence of intra-amniotic infection. Our data are consistent with the working hypothesis that placental insufficiency may be a cause of idiopathic spontaneous PTB.

The placental samples used for this analysis were derived from women with threatened PTB who participated in a tightly controlled multicenter clinical trial comparing the effects of single versus multiple courses of antenatal steroids. Sawady et al. [34] had reported that placental weight may be affected by repeated steroid therapy, but not placental histologic features in this case series [34]. This finding differed from Ghidini et al. [35], who showed an association between the increased number of steroid doses and villous fibrosis [35]. Regardless, even if antenatal steroids do affect placental histology, there was no difference in the frequency of single versus multiple doses between our three PTB study groups (idiopathic, histologic infection, and other); so, this potential confounder is less likely to influence our data.

Given the significance of intra-amniotic infection in PTB, it is important to point out that the frequency of acute chorioamnionitis in our study was within the reported range of 15-60\% [2-4]. Most of the cases positive for acute chorioamnionitis in our study delivered before 34 weeks' gestation, which is similar to the results by Watts et al. [36] who reported an inverted relationship between positive amniotic fluid cultures and gestational age [36].

PVH was common in idiopathic PTB (84\% of cases) and it was more likely to occur after 34 weeks gestation. Other causes of PTB such as preeclampsia and IUGR were also more likely to deliver later in gestation in our study and had a similarly high frequency of PVH (89\%). It is therefore possible that the pathologic diagnosis of PVH may be biased the closer delivery is to 37 weeks. This potential bias has been studied by Khong et al. [35] who have shown that the magnitude and direction of error when assessing placental maturity is actually less after 31 weeks gestation than earlier, with a relative underestimation of gestational age in later preterm deliveries [31]. Gestational age was tightly controlled in our case series [33] 
and the pathologist's diagnosis was made while blinded to gestational age, which is important to reduce bias [31].

Notably, we did not observe distal villous hypoplasia in our series, which has been associated with severe early-onset IUGR, but not isolated preeclampsia [12]. There were six cases of IUGR in our series, but none showed distal villous hypoplasia as defined by Kaufmann's group [12] or the Society for Pediatric Pathology [29]. The absence of distal villous hypoplasia in idiopathic PTB suggests that similar to preeclampsia and later onset IUGR, the mechanism may be related to oxidative stress rather than developmental pathology [10-12].

We defined PVH as premature maturation of terminal chorionic villi (resembling term, greater than 38 weeks gestation) with conspicuous syncytial knotting (Tenney- Parker changes) in preterm placentas. Our specific definition requiring that the PVH diagnosis be made only in preterm deliveries should make this study more reproducible, because pathologists can reliably distinguish mature term from preterm villous architecture [32]. This is important because although others have reported significant differences in placental villous architecture associated with PTB [25-30], the reproducibility of these associations has been questioned [31]. Our strict preterm definition, together with our evaluation for distal villous hypoplasia, and the strength of our tightly controlled multicenter trial, reinforces prior studies from the 1990s [20,27], suggesting that placental insufficiency may cause idiopathic PTB. Moreover, emphasizing the potential significance of the role of placental insufficiency in idiopathic PTB is timely provided differences in blood flow $[23,24]$, novel genetic signatures [5] and serum proteomics [6].

We propose that idiopathic PTB may be part of a continuum of placental insufficiency phenotypes in the spectrum of preeclampsia and IUGR. Terminal villous formation accelerates exponentially in the third trimester of pregnancy so that by term the placental surface area available for nutrient exchange reaches $13 \mathrm{~m}^{2}$ [37]. This increase in the number of terminal villi and surface area is important to nourish the rapidly growing fetus. Indeed, terminal villous differentiation appears to accommodate the 10 -fold increase in fetal weight from 20 weeks gestation to term [12]. If we accept the hypothesis that there may be abnormal uteroplacental blood flow leading to oxidative stress in preeclampsia, late onset IUGR, and some cases of spontaneous PTB, then PVH may be either a compensatory mechanism to increase nutrient transfer surface area, or it may be a histologic marker of oxidative damage. We did not measure surface area in our study and this metric was not required for a diagnosis of PVH. However, future studies could test this hypothesis by directly comparing uteroplacental blood flow with placental stereometry [37] and pregnancy outcomes.

The shared mechanism leading to abnormal uteroplacental blood flow may be abnormal spiral artery remodeling. Pregnancy-induced vascular remodeling of the uterine spiral arteries is key to a successful pregnancy [13-15] and there are numerous reports correlating abnormal remodeling with PTB [18-22]. Variations in spiral artery remodeling between women may be related to differences in placental invasion, or differences in maternally mediated responses [10]. Significant variations in remodeling may be detected as early as 
the first trimester [38,39] and they are maintained until term [17]. Starzyk et al. (1997) reported using placental bed biopsies that there is a distribution of spiral artery changes from normal to abnormal independent of pregnancy outcome [17]. However, the distribution is weighted toward more and smaller-caliber thick-walled spiral arteries in women with preeclampsia compared with normal controls [17]. Variations in spiral artery remodeling within and between women raise the possibility that various pregnancy complications associated with placental insufficiency may be pleiotropic manifestations of a shared underlying process.

The weaknesses of our study include a modest sample size and the absence of culture/PCR data to confirm the presence of intra-amniotic infection. Our retrospective analysis was dependent on available histologic sections from a tightly controlled multicenter clinical trial designed to test the effects of antenatal steroids in women with threatened PTB [33]. Although the sample size is modest, the strength of this study is strict inclusion/exclusion criteria and uniform sample collection. It was a randomized multicenter trial, which addresses potential selection bias related to patient populations and sample collection. Unfortunately, placental weight measurements were not obtained, but it is important to recognize that small for gestational age placentas are associated with IUGR and not spontaneous PTB [26].

In conclusion, our data suggest a potential relationship between PVH and idiopathic PTB. The precise role of placental insufficiency in the development of idiopathic preterm labor in humans remains unknown, but this hypothesis may be tested once methods are developed to accurately measure placental blood flow in women.

\section{Acknowledgments}

We are very grateful for the diligence and organization provided by Dr Michael Varner (Ob/Gyn, University of Utah), Dr Kenneth Ward (Juneau Biosciences, Utah) and Lesa Nelson (Taueret Laboratories, Utah) to maintain the tissue bank from the NICHD Maternal-Fetal Medicine Units (MFMU) Network antenatal corticosteroids trial for our study. We also wish to thank the following subcommittee members who participated in protocol development and coordination between clinical research centers (Michelle DiVito, MSN and Francee Johnson, RN, BSN), protocol/ data management and statistical analysis (Elizabeth Thom, Ph.D.). In addition to the authors, other members of the Eunice Kennedy Shriver National Institute of Child Health and Human Development MaternalFetal Medicine Units Network are as follows:

Drexel University - M. DiVito, A. Sciscione, V. Berghella, M. Pollock, M. Talucci

Wayne State University - M. Dombrowski, G. Norman, A. Millinder, C. Sudz, D. Driscoll

The Ohio State University - F. Johnson, J. Iams, M. Landon, S. Meadows, P. Shubert

University of Utah - M. Varner, K. Anderson, A. Guzman, A. Crowley, M. Fuller

Northwestern University - G. Mallett

University of Texas Southwestern Medical Center - K. Leveno, D. Weightman, L. Fay-Randall, P. Mesa

Wake Forest University Health Sciences - M. Harper, P. Meis, M. Swain, C. Moorefield

University of Pittsburgh - S. Caritis, T. Kamon, K. Lain, M. Cotroneo

Columbia University - M. Miodovnik, F. Malone, V. Pemberton, S. Bousleiman

Case Western Reserve University-MetroHealth Medical Center - P. Catalano, C. Milluzzi, C. Santori

University of North Carolina at Chapel Hill - K. Moise, K. Dorman

University of Chicago - A. Moawad, P. Jones, G. Mallett

J Matern Fetal Neonatal Med. Author manuscript; available in PMC 2014 May 05. 
University of Miami - D. Martin, F. Doyle

The University of Texas Health Science Center at Houston - L. Gilstrap, M.C. Day

Brown University - M. Carpenter, D. Allard, J. Tillinghast

University of Alabama at Birmingham - A. Northen, K. Bailey

University of Cincinnati - M. Miodovnik, H. How, N. Elder, B. Alexander, W. Girdler

University of Tennessee - B. Mabie, R. Ramsey

Eunice Kennedy Shriver National Institute of Child Health and Human Development - D. McNellis, K. Howell, S. Tolivaisa

The George Washington University Biostatistics Center - E.Thom, F. Galbis-Reig, L. Leuchtenburg MFMU Network Steering Committee Chair (Vanderbilt University Medical Center) - S. Gabbe

Dr Morgan was funded by the Office of Research on Women's Health and the Eunice Kennedy Shriver National Institute of Child Health and Human Development, Oregon BIRCWH: HD043488-08. This research was also supported by grants from the Eunice Kennedy Shriver National Institute of Child Health and Human Development (NICHD) [HD21410, HD21414, HD27869, HD27917, HD27905, HD27860, HD27861, HD27915, HD34122, HD34116, HD34208, HD34136, HD40500, HD40485, HD40544, HD40545, HD40560, HD40512, HD36801] and M01-RR-000080 from the National Center for Research Resources (NCRR) and its content is solely the responsibility of the authors and does not necessarily represent the official views of the NICHD, the National Institutes of Health and the NCRR.

\section{References}

1. Kramer MS, Demissie K, Yang H, et al. The contribution of mild and moderate preterm birth to infant mortality. Fetal and Infant Health Study Group of the Canadian Perinatal Surveillance System. JAMA. 2000; 284:843-849. [PubMed: 10938173]

2. DiGiulio DB, Romero R, Amogan HP, et al. Microbial prevalence, diversity and abundance in amniotic fluid during preterm labor: a molecular and culture-based investigation. PLoS One. 2008; 3:e3056. [PubMed: 18725970]

3. Hillier SL, Martius J, Krohn M, et al. A case-control study of chorioamnionic infection and histologic chorioamnionitis in prematurity. N Engl J Med. 1988; 319:972-978. [PubMed: 3262199]

4. Romero R, Salafia CM, Athanassiadis AP, et al. The relationship between acute inflammatory lesions of the preterm placenta and amniotic fluid microbiology. Am J Obstet Gynecol. 1992; 166:1382-1388. [PubMed: 1595794]

5. DeFranco E, Teramo K, Muglia L. Genetic influences on preterm birth. Semin Reprod Med. 2007; 25:40-51. [PubMed: 17205422]

6. Pereira L, Reddy AP, Alexander AL, et al. Insights into the multifactorial nature of preterm birth: proteomic profiling of the maternal serum glycoproteome and maternal serum peptidome among women in preterm labor. Am J Obstet Gynecol. 2010; 202:555.e1-555.e10. [PubMed: 20413102]

7. Hollier LM. Preventing preterm birth: what works, what doesn't. Obstet Gynecol Surv. 2005; 60:124-131. [PubMed: 15671901]

8. Fox, H. Pathology of the placenta. London, Philadelphia, Toronto: W.B. Saunders Company Ltd; 1978.

9. Assali N, Nuwayhid B, Brinkman C. Placental insufficiency: problems of etiology, diagnosis and management. Eur J Obstet Gynecol Reprod Biol. 1975; 5:87-91. [PubMed: 233751]

10. Burton G, Woods A, Jauniaux E, et al. Rheological and physiological consequences of conversion of the maternal spiral arteries for uteroplacental blood flow during human pregnancy. Placenta. 2009; 30:473-482. [PubMed: 19375795]

11. Mayhew T, Barker B. Villous trophoblast: morphometric perspectives on growth, differentiation, turnover and deposition of fibrin-type fibrinoid during gestation. Placenta. 2001; 22:628-638. [PubMed: 11504531]

12. Kingdom J, Huppertz B, Seaward G, et al. Development of the placental villous tree and its consequence for fetal growth. Eur J Obstet Gynecol Reprod Biol. 2000; 92:35-43. [PubMed: 10986432] 
13. Brosens I, Robertson W, Dixon H. The physiological response of the vessels of the placental bed to normal pregnancy. J Pathol Bacteriol. 1967; 93:569-579. [PubMed: 6054057]

14. Brosens I, Robertson W, Dixon H. The role of the spiral arteries in the pathogenesis of preeclampsia. Obstet Gynecol Annu. 1972; 1:177-191. [PubMed: 4669123]

15. Pijnenborg R, Dixon G, Robertson W, et al. Trophoblastic invasion of human decidua from 8 to 18 weeks of pregnancy. Placenta. 1980; 1:3-19. [PubMed: 7443635]

16. Morgan T, Craven C, Lalouel J-M, et al. Angiotensinogen Thr235 variant is associated with abnormal physiologic change of the uterine spiral arteries in first trimester decidua. Am J Obstet Gynecol. 1999; 180:95-102. [PubMed: 9914585]

17. Starzyk K, Salafia C, Pezzullo J, et al. Quantitative differences in arterial morphology define the placental bed in preeclampsia. Hum Pathol. 1997; 28:353-358. [PubMed: 9042801]

18. Khong TY. Placental vascular development and neonatal outcome. Semin Neonatol. 2004; 9:255263. [PubMed: 15251142]

19. Kelly R, Holzman C, Senagore P, et al. Placental vascular pathology findings and pathways to preterm delivery. Am J Epidemiol. 2009; 170:148-158. [PubMed: 19509320]

20. Arias F, Rodriquez L, Rayne SC, et al. Maternal placental vasculopathy and infection: two distinct subgroups among patients with preterm labor and preterm ruptured membranes. Am J Obstet Gynecol. 1993; 168:585-591. [PubMed: 8438933]

21. Kim YM, Chaiworapongsa T, Gomez R, et al. Failure of physiologic transformation of the spiral arteries in the placental bed in preterm premature rupture of membranes. Am J Obstet Gynecol. 2002; 187:1137-1142. [PubMed: 12439491]

22. Ogunyemi D, Murillo M, Jackson U, et al. The relationship between placental histopathology findings and perinatal outcome in preterm infants. J Matern Fetal Neonatal Med. 2003; 13:102109. [PubMed: 12735410]

23. Strigini FA, Lencioni G, De Luca G, et al. Uterine artery velocimetry and spontaneous preterm delivery. Obstet Gynecol. 1995; 85:374-377. [PubMed: 7862375]

24. Misra VK, Hobel CJ, Sing CF. Placental blood flow and the risk of preterm delivery. Placenta. 2009; 30:619-624. [PubMed: 19464055]

25. Salafia CM, Pezzullo JC, Lopez-Zeno JA, et al. Placental pathologic features of preterm preeclampsia. Am J Obstet Gynecol. 1995; 173:1097-1105. [PubMed: 7485300]

26. Roberts DJ, Post MD. The placenta in pre-eclampsia and intrauterine growth restriction. J Clin Pathol. 2008; 61:1254-1260. [PubMed: 18641412]

27. Salafia CM, Vogel CA, Vintzileos AM, et al. Placental pathologic findings in preterm birth. Am J Obstet Gynecol. 1991; 165:934-938. [PubMed: 1951558]

28. Langston C, Kaplan C, Macpherson T, et al. Practice guideline for examination of the placenta: developed by the Placental Pathology Practice Guideline Development Task Force of the College of American Pathologists. Arch Pathol Lab Med. 1997; 121:449-476. [PubMed: 9167599]

29. Redline RW, Boyd T, Campbell V, et al. Maternal vascular underperfusion: nosology and reproducibility of placental reaction patterns. Pediatr Dev Pathol. 2004; 7:237-249. [PubMed: 15022063]

30. Benirschke, P.; Kaufmann, P. Pathology of the human placenta. Berlin: Springer; 2000.

31. Khong TY, Staples A, Bendon RW, et al. Observer reliability in assessing placental maturity by histology. J Clin Pathol. 1995; 48:420-423. [PubMed: 7629287]

32. Grether JK, Eaton A, Redline RW, et al. Reliability of placental histology using archived specimens. Paediatr Perinatol Epidemiol. 1999; 13:489-495.

33. Wapner RJ, Sorokin Y, Thom EA, et al. for the National Institute of Child Health and Human Development Maternal Fetal Medicine Units Network. Single versus weekly courses of antenatal corticosteroids: evaluation of safety and efficacy. Am J Obstet Gynecol. 2006; 195:633-642. [PubMed: 16846587]

34. Sawady J, Mercer BM, Wapner RJ, et al. for the National Institute of Child Health and Human Development Maternal Fetal Medicine Units Network. Beneficial effects of antenatal repeated steroids study: impact of repeated doses of antenatal corticosteroids on placental growth and histologic findings. Am J Obstet Gynecol. 2007; 197:281.e1-281.e8. [PubMed: 17826421] 
35. Ghidini A, Pezzullo JC, Sylvestre G, et al. Antenatal corticosteroids and placental histology in preterm birth. Placenta. 2001; 22:412-417. [PubMed: 11373151]

36. Watts DH, Krohn MA, Hillier SL, et al. The association of occult amniotic fluid infection with gestational age and neonatal outcome among women in preterm labor. Obstet Gynecol. 1992; 79:351-357. [PubMed: 1738513]

37. Luckhardt M, Leiser R, Kingdom J, et al. Effect of physiologic perfusion-fixation on the morphometrically evaluated dimensions of the term placental cotyledon. J Soc Gynecol Invest. 1996; 3:166-171.

38. Morgan T, Craven C, Nelson L, et al. Angiotensinogen T235 expression is elevated in decidual spiral arteries. J Clin Invest. 1997; 100:1406-1415. [PubMed: 9294106]

39. Craven C, Morgan T, Ward K. Decidual spiral artery remodeling begins before cellular interaction with trophoblasts. Placenta. 1998; 19:241-252. [PubMed: 9639319] 
(A)

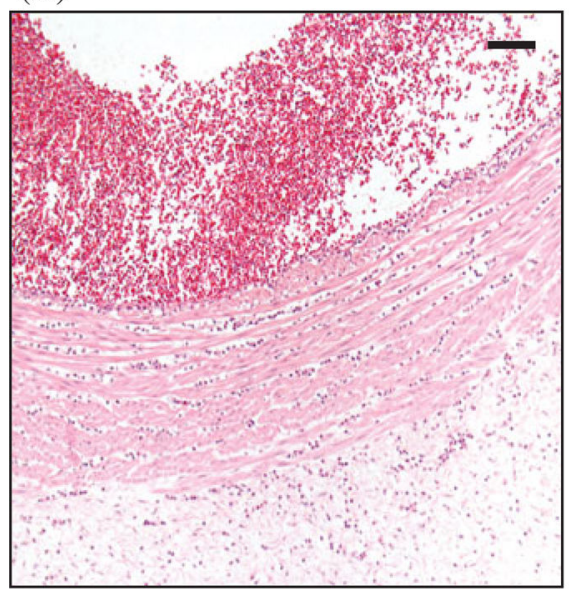

(D)

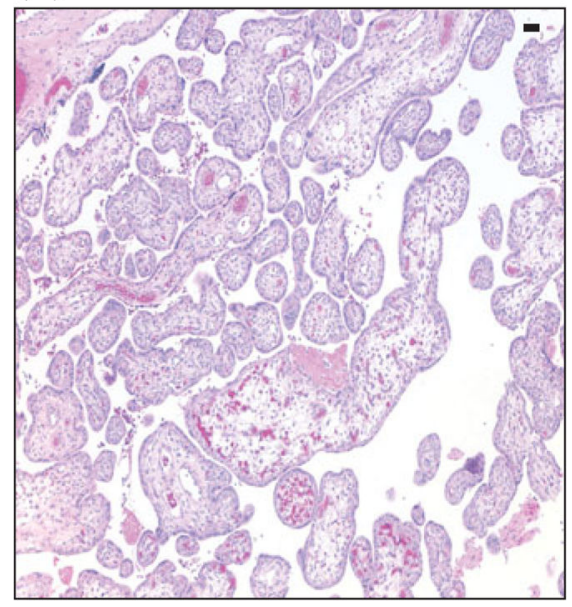

(B)

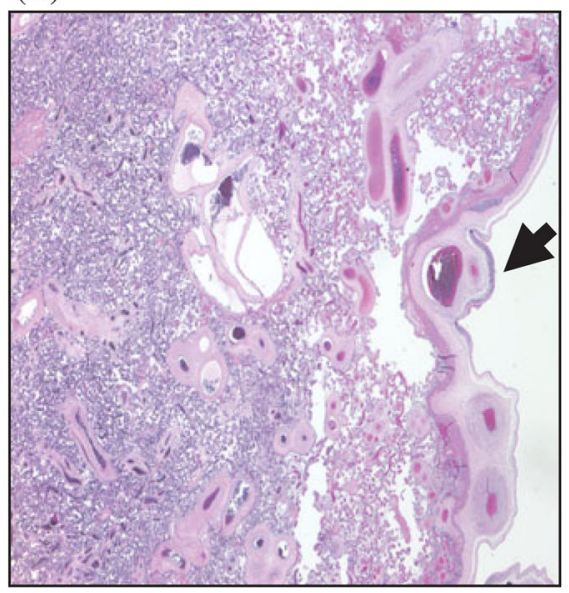

(E)

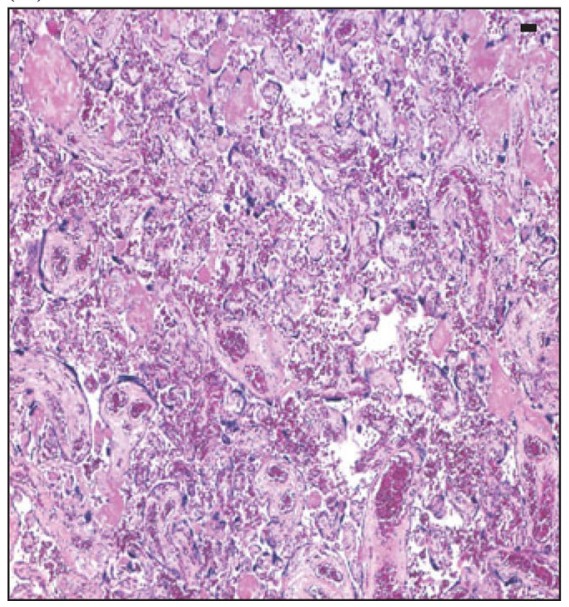

(C)

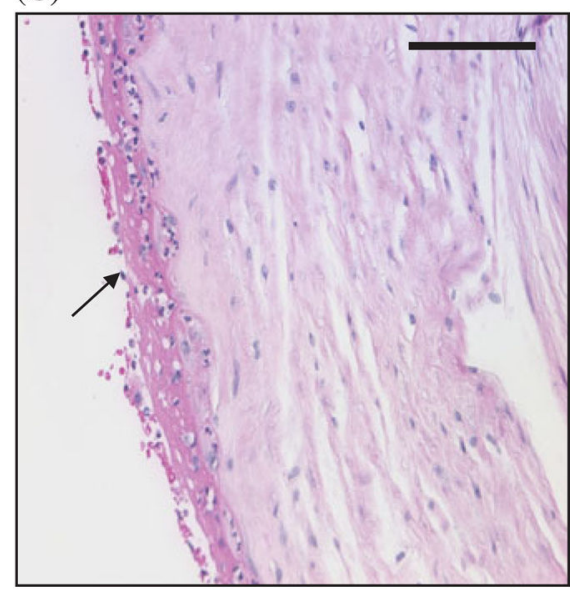

(F)

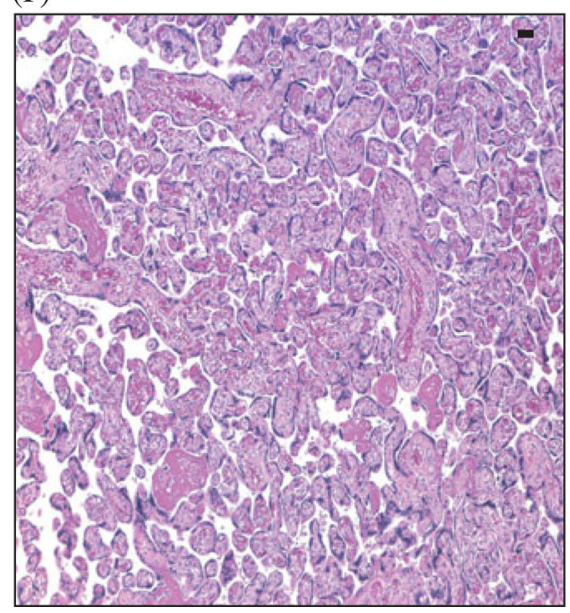

Figure 1.

Histologic features of intra-amniotic infection and placental insufficiency. (A) Funisitis is a specific indicator of infection and is defined by the presence of fetal neutrophils marginating into the blood vessel wall. (B-C) Acute chorioamnionitis is a sensitive sign of infection and is defined by maternal neutrophils marginating into the placental chorionic plate. $\mathrm{PVH}$ is characterized by term-appearing terminal chorionic villi with conspicuous syncytial knotting in preterm samples. (D) Normal villous maturation before 37 weeks gestation (this case is at 32 weeks) shows a predominance of intermediate villi with fewer numbers of terminal villi and only inconspicuous perivillous fibrin and syncytial knots. (E) In contast, PVH is readily diagnosed in many cases of idiopathic PTB (this case too was at 32 weeks), because the chorionic villi resemble preterm preeclampsia, IUGR or placental sections from term (F). Bar is $100 \mu \mathrm{m}(\mathrm{A}-\mathrm{F})$. 


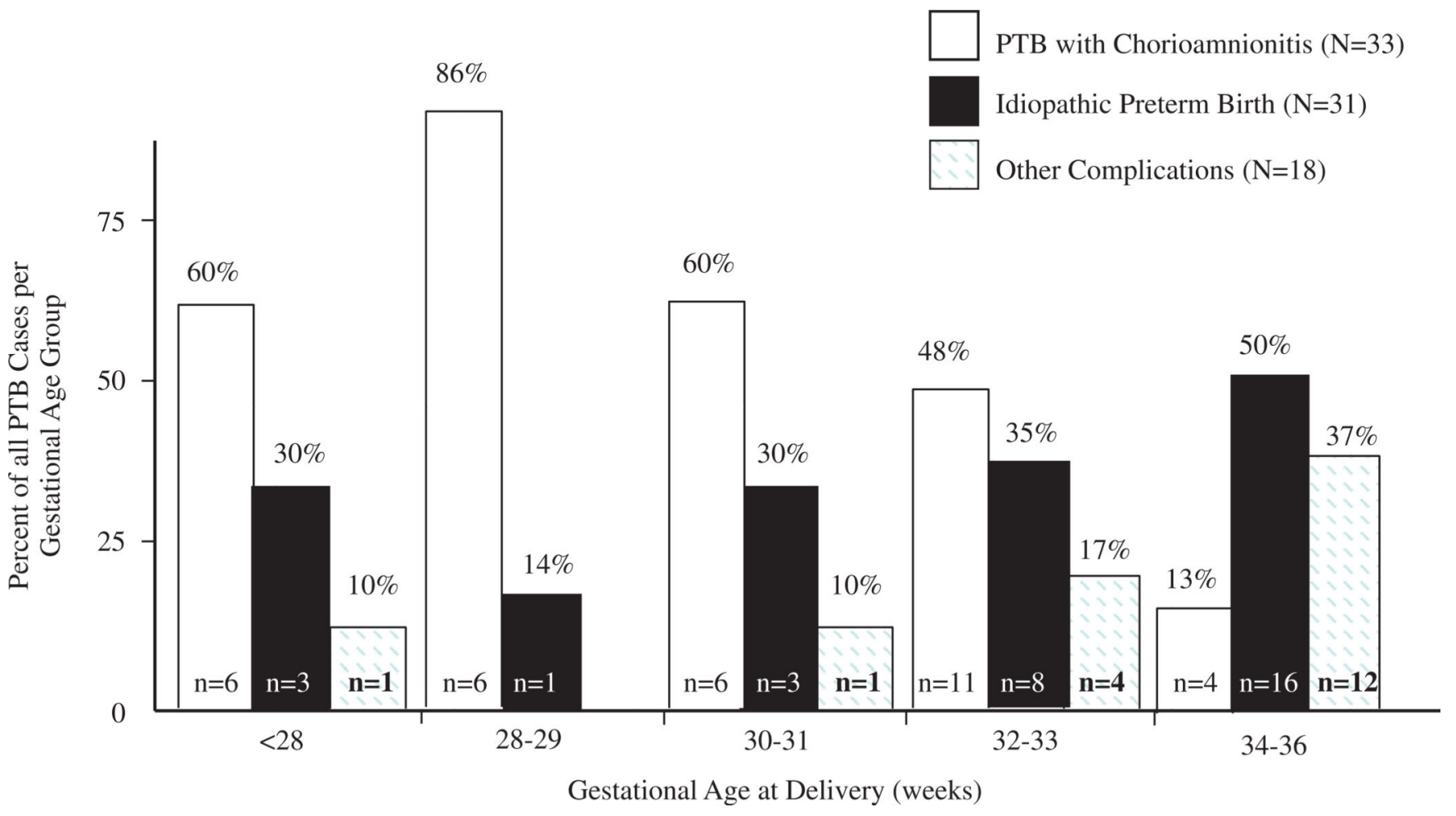

Figure 2.

PVH in PTB related to gestational age. The frequency of acute chorioamnionitis (empty bars), idiopathic PTB (black filled in bars), and other known covariates (e.g. preeclampsia/gestational hypertension, IUGR, abruption, PPROM, diabetes) suggests infection may be the most common cause of PTB before 32 weeks and less common after 34 weeks. 


\section{Table 1}

$\mathrm{PVH}$ is Common in Idiopathic PTB.

\begin{tabular}{lccc}
\hline & $\begin{array}{c}\text { Acute } \\
\text { chorioamnionitis } \\
(\boldsymbol{n}=\mathbf{3 3})\end{array}$ & $\begin{array}{c}\text { Other } \\
\text { complications } \\
(\boldsymbol{n}=\mathbf{1 8})\end{array}$ & $\begin{array}{c}\text { Idiopathic } \\
\mathbf{P T B} \\
(\boldsymbol{n}=\mathbf{3 1})\end{array}$ \\
\hline Normal villous maturation & $23 / 33(70 \%)$ & $2 / 18(11 \%)$ & $5 / 31(16 \%)$ \\
PVH & $10 / 33(30 \%)$ & $16 / 18(89 \%)$ & $26 / 31(84 \%)$ \\
\hline
\end{tabular}

Compared to PTB with histologic evidence of infection, idiopathic PTB had significantly more PVH, even when controlled for covariants such as gestational age, steroid treatment group, and other known causes of PTB (preeclampsia/gestational hypertension, IUGR, PPROM without infection, abruption, diabetes)

${ }^{*}<0.001$. 